\title{
Evaluation of the anatomical measurements of the temporomandibular joint by cone-beam computed tomography
}

\author{
M. Ocak ${ }^{1}$, M.F. Sargon², K. Orhan ${ }^{3}$, B. Bilecenoğlu' ${ }^{1}$, F. Geneci' ${ }^{4}$, M.B. Uzuner \\ ${ }^{1}$ Deparment of Anatomy, Faculty of Dentistry, Ankara University, Ankara, Turkey \\ 2Department of Anatomy, Faculty of Medicine, Atilim University, Ankara, Turkey \\ ${ }^{3}$ Deparment of Dentomaxillofacial Radiology, Faculty of Dentistry, Ankara University, Ankara, Turkey \\ ${ }^{4}$ Department of Anatomy, Faculty of Medicine, Yıldırım Beyazıt University, Ankara, Turkey \\ ${ }^{5}$ Department of Anatomy, Faculty of Medicine, Kafkas University, Kars, Turkey
}

[Received: 25 April 2018; Accepted: 19 June 2018]

\begin{abstract}
Background: To examine the detailed anatomy of the normal temporomandibular joint (TMJ) in a large series of patients divided into different age groups.

Materials and methods: Cone-beam computed tomography images of 100 patients included in the study. Morphometric analysis regarding mandibular condyle and mandibular fossa, articular tubercle and the zygomatic arch was done. The volumetric and surface measurements of mandibular condyles (total tissue volume [TV], total bone volume [BV], bone surface area [BS] and percentage of bony tissue of the mandibular condyle [BV/TV]) were also measured.

Results: Statistical analysis was performed and statistically significant differences according to the side of the joint, sex, and age groups were reported. Additionally, correlations between aging and all of these parameters were also determined. Conclusions: TV, BV, BS and BV/TV parameters according to side, age, and sex groups were defined for normal TMJ which may help to understand the onset and progress of TMJ disorders. (Folia Morphol 2019; 78, 1: 174-181)
\end{abstract}

Key words: temporomandibular joint, cone-beam computed tomography, sex, age, anatomy

\section{INTRODUCTION}

The temporomandibular joint (TMJ) is a mobile joint used during speech, mastication, and swallowing. The joints on both sides form a functional unit and they work synchronically. This joint lies in between the condylar process of the mandible and mandibular fossa (glenoid fossa) and the articular tubercle of the temporal bone $[9,10]$. The TMJ is a complex joint because of its anatomical and histological differences from other joints and its specific biomechanical characteristics [14]. The joint's surface is wholly convex in the anteroposterior direction. However, it shows a mild convexity in the mediolateral direction. Additionally, the condylar process may have different shapes and variations $[6,10]$. The mandibular fossa has a concave shape and its joint surface is 3-4 times larger than the head of the mandible. The degree of convexity of the articular tubercle is variable, and it has an important role in the anterior movement of the mandible $[6,10]$.

In the literature, there are many studies on the anatomy, histology, and biomechanics of the TMJ. However, there are only a few studies in the literature describing the morphometry of the joint in an anatomically normal population [7]. 
The specific characteristics of the TMJ according to age, sex, and position of the joint in anatomically normal patients are important clinically. In this study, the authors aimed to examine the detailed morphometry of TMJ in anatomically normal patients, which will play an important role in understanding the aetiology of joint disorders, the effects of surgical procedures in this joint, the effects of diseases on TMJ, the effects of surgical interventions in the head and neck region, and age-related changes in TMJ.

\section{MATERIALS AND METHODS}

\section{Study design}

The study was performed in the Department of Anatomy, Faculty of Medicine, Hacettepe University, Ankara, Turkey by using the cone-beam computed tomography (CBCT) images of 100 anatomically normal patients. The patients' data were obtained from the Department of Dentomaxillofacial Radiology, Faculty of Dentistry, Ankara University, Ankara, Turkey. All the patients had a normal TMJ structure radiologically, and they visited the Radiology Department between 2012 and 2017. All proposed subjects agreed to participate in the study, and all provided written informed consent. Ethical approval for the study was obtained from University Ethics Committee (GO 16/168-08). The study included 50 male and 50 female patients. The patients' ages varied between 20 and 69 years (mean age $44 \pm 14.403$ ). The patients were divided into five groups, and each group included 10 patients. The age groups of male and female patients were 20-29, 30-39, 40-49, 50-59, and $60-69$ years. The study population comprised 100 subjects: 50 (50\%) females, $50(50 \%)$ males, who had undergone $\mathrm{CBCT}$ imaging for pre-evaluation of impacted third molar and impacted teeth surgery.

\section{CBCT protocol}

The patients' images were taken in the supine position using the standard protocol of CBCT (Planmeca Promax 3D Max). While obtaining the images, the mandible was in a closed position and the patient was immobile. The images were recorded at $96 \mathrm{kVp}$ and $10 \mathrm{~mA}, 575 \times 575 \mathrm{~mm}$ image plane. The section thickness was $0.4 \mathrm{~mm}$ and they were recorded in isotropic $400 \mu \mathrm{m}$ voxel. The images were obtained from the level of fifth cervical vertebra up to the upper border of the orbit. All the images were of $0.4 \mathrm{~mm}$ thickness and they were obtained in coronal, axial, and sagittal planes. Reconstructions and measurements were per- formed with a 21.3-inch flat panel and a TFT medical screen having active a colour matrix (NEC MultiSync MD215MG, Munchen, Germany) of $2048 \times 2560$ pixels, $75 \mathrm{~Hz}$ and $0.17 \mathrm{~mm}$ point character frequency of 11.9 bit. While obtaining the relation between the mandibular fossa, articular tubercle, and mandibular condyle, Romexis-Viewer (ver. 3.2.0, Planmeca) software was used. For the measurements of the volume and bone surface areas of mandibular condyles (MCA), CT-analyser (ver. 1.12.9, Skyscan) software was used. All the measurements of the study were performed by two researchers in different periods and each researcher took the measurements two times. There was a gap of 2 weeks, between the measurements taken by both the researchers. The statistical analysis of harmony of the two researchers was also determined.

\section{Measurements obtained from coronal sections}

The images were calibrated by finding the midline of the odontoid process in the vertical axis, and both the TMJs were put into equal angulations in the axial plane. The measurements were performed using images in which the MCA were largest in posteroanterior coronal images. In coronal sections, the width of mandibular condyle, the distance between the mandibular fossa and medial end of mandibular condyle, the distance between the mandibular fossa and lateral end of mandibular condyle, and the distance between the mandibular fossa and most upper point of mandibular condyle were measured. For angular measurements, the angle between the long axis of the mandibular condyle and horizontal axis, the angle between the long axis of mandibular ramus and vertical axis, and the angle between the long axis of the mandibular condyle and the long axis of the ramus of the mandible were measured (Fig. 1).

The width of the mandibular condyle (MCW) was measured in the coronal section in which the head of the condyle was observed as being the largest. In this measurement, the distance between the medial and lateral ends of the head was measured. For the measurement of the distances between the mandibular fossa and medial end of the mandibular condyle (CONM-MF) or lateral end of the mandibular condyle (CONL-MF), a vertical line was drawn toward the mandibular fossa. Additionally, the least distance between the mandibular condyle and mandibular fossa in the coronal plane (CONT-MF(C)) was calculated.

The angle in between the long axis of the mandibular condyle and the horizontal plane (CLA-HA) was 


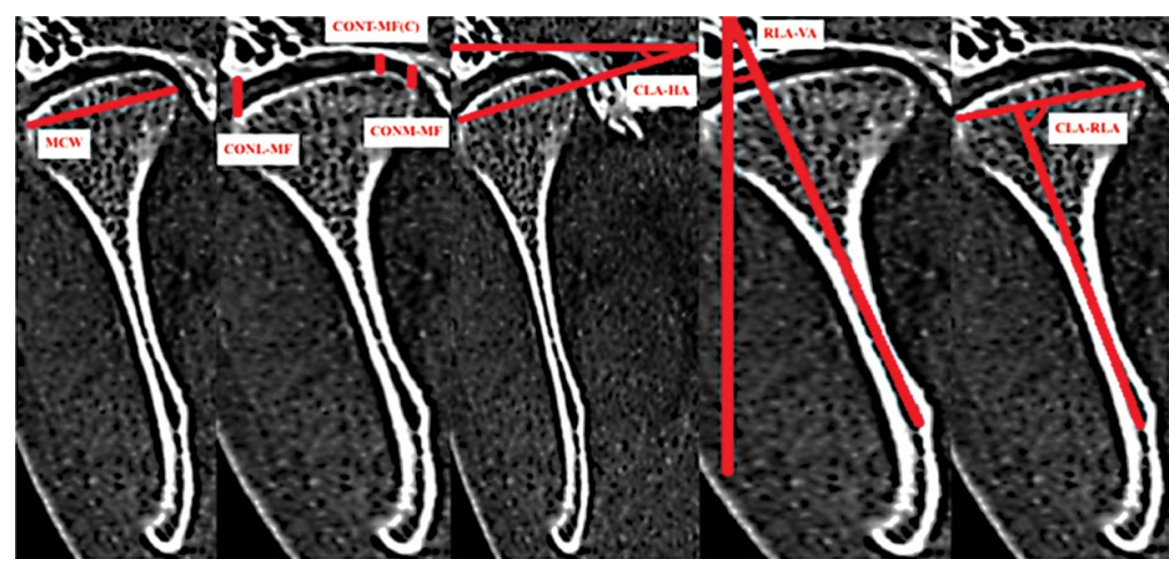

Figure 1. Measurements obtained from coronal sections; MCW — the width of the mandibular condyle; CONL-MF — the distance in between the mandibular fossa and medial end of the mandibular condyle; CONT-MF(C) - the measurement of the least distance in between the mandibular condyle and mandibular fossa in the coronal plane; CONM-MF — the distance in lateral end of the mandibular condyle, CLA-HA — the angle in between the long axis of mandibular condyle and horizontal plane; RLA-VA — measuring the angle in between the long axis of ramus of mandible and vertical plane; CLA-RLA — the angle in between the long axis of mandibular condyle and long axis of ramus of mandible.

measured in the section in which the condyle was observed as the largest. While measuring the angle in between the long axis of the ramus of the mandible and vertical plane (RLA-VA), the line drawn from the posterior border of the ramus of the mandible was accepted to be the long axis of the ramus. The angle between the long axis of the mandibular condyle and long axis of the ramus of mandible (CLA-RLA) was also calculated.

\section{Measurements of sagittal sections}

The measurements were done using the widest images of mandibular condyle obtained in lateral to medial observations in the anteroposterior direction. The measurements obtained in sagittal sections were the distance between the mandibular fossa and tip of the mandibular condyle, the distance between the tip of the mandibular condyle and mid-point of mandibular condyle, and the distance between the mid-point of the mandibular condyle and mandibular fossa. Additionally, the curvature of the articular tubercle was also measured.

For the measurement of the distance between the mandibular fossa and tip of the mandibular condyle from the sagital plane (CONT-MF(S)), the smallest distance between these two points was accepted as the tip point of the mandibular condyle. For the measurement of the distance between a vertical line was drawn through mandibular fossa from the tip point for measurements. For the measurement of the distance between the mid-point of mandibu- lar condyle and tip of mandibular condyle (CONMi-CONT), the mid-point of the mandibular condyle was obtained in the mediolateral direction and the distance between this point and tip of the mandibular condyle was measured. For the measurement of the distance between the mid-point of the mandibular condyle and mandibular fossa (CONMi-MF), a vertical line was drawn through the mandibular fossa and measurements were calculated. The curvature of the articular tubercle (AT Tilt) was obtained from the angle between the tangential line drawn to the posterior border of the TMJ and Frankfurt horizontal plane [4].

\section{Axial section measurements}

In axial sections, the measurements were done using the images in which the anteroposterior diameters of the MCA of both sides were seen the largest. For the measurement of the angle in between the MCA of both sides, the two lines drawn parallel to the anteroposterior diameters of the two condyles were joined with each other in the mid-sagittal line and the angle was calculated (Fig. 2).

\section{Measurements by 3D remodelling of the bone}

Three-dimensional (3D) remodelling was done using the images in which the articular tubercle, zygomatic arch, and mandibular fossa were observed in the maximal clearance. By this method, the height of the articular tubercle, the distance between the articular tubercle and mandibular fossa, and the angle between the long axis of the articular tuber- 


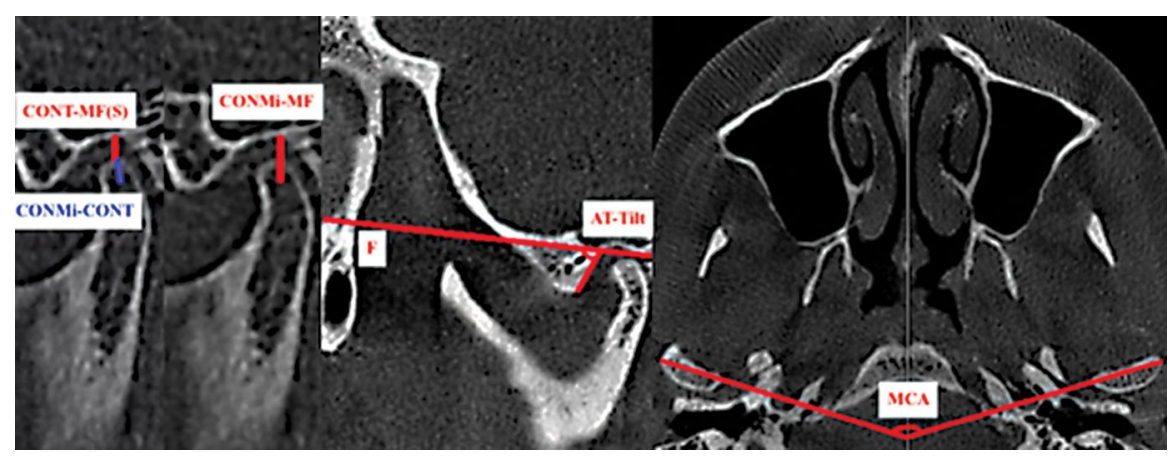

Figure 2. Measurements of sagittal and axial sections. CONT-MF(S) - the measurement of distance in between the mandibular fossa and tip of the mandibular condyle; CONMi-CONT - the measurement of the distance in between the mid-point of mandibular condyle and tip of mandibular condyle; CONMi-MF — measurement of the distance in between the mid-point of mandibular condyle and mandibular fossa; $\mathrm{F}$ - Frankfurt horizontal plane; AT-Tilt — the curvature of the articular tubercle; MCA — for the measurement of the angle in between the mandibular condyles.

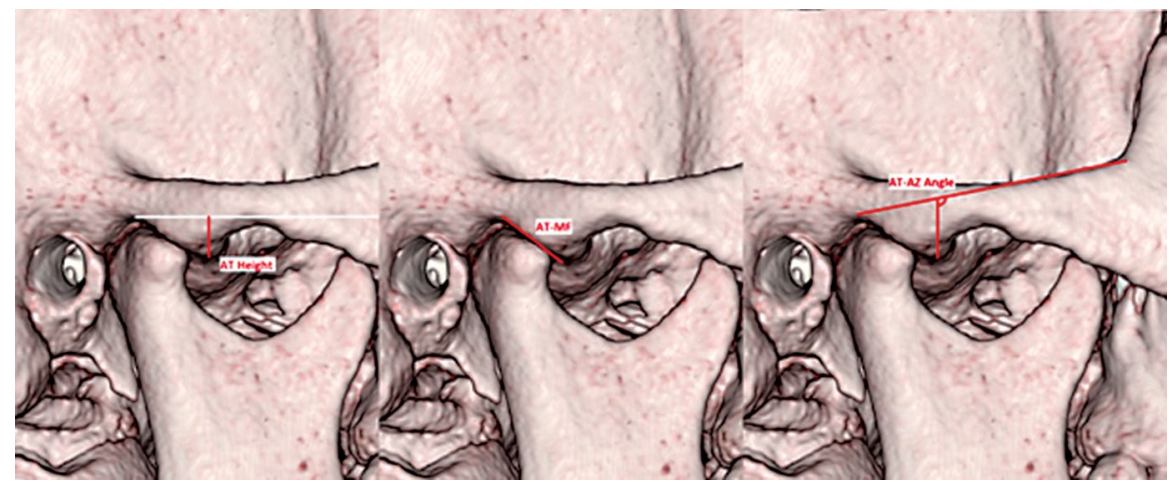

Figure 3. Measurements by three-dimensional remodelling of the bone; AT-Height - the height of the articular tubercle; AT-MF — the distance in between the articular tubercle and mandibular fossa; AT-ZA Angle — the angle in between the long axis of articular tubercle and transverse axis of zygomatic arch.

cle and transverse axis of the zygomatic arch were measured.

The height of the articular tubercle (AT Height) and the distance between the articular tubercle and mandibular fossa (AT-MF) were also measured. For the measurement of the AT-MF, the distance between the deepest part of mandibular fossa and the most inferior point of articular tubercle was calculated. The angle in between the long axis of the articular tubercle and transverse axis of zygomatic arch (AT-ZA angle) was found by drawing a tangential line to the upper border of the zygomatic arch and intersecting this line with the mid-point of the base of the articular tubercle (Fig. 3).

\section{Measurements of the bony tissues belonging to MCA}

The volumetric and surface measurements of the bony tissues belonging to MCA were done using CTAn
Table 1. Angle between mandibular condyles (MCA)

\begin{tabular}{cccc}
\hline & $\begin{array}{c}\text { Mean } \pm \text { standard } \\
\text { deviation }\end{array}$ & $\begin{array}{c}\text { 95\% confidence } \\
\text { interval }\end{array}$ & $\begin{array}{c}\text { Minimum- } \\
\text {-Maximum }\end{array}$ \\
\hline $\left.\mathrm{MCA}^{\circ}\right]$ & $135.51 \pm 15.23$ & $132.49-138.54$ & $94.49-168.93$ \\
\hline
\end{tabular}

(ver. 1.12.9, Skyscan) software programme. From this programme, the images were transferred to computers and in necessary cases 3D modelling was also performed [4]. Bony tissues belonging to MCA were determined in the axial images and the regions of interest (ROls) were chosen. Then, thresholding was done. In the selected $\mathrm{ROI}$ areas, the total tissue volume of mandibular condyle (TV), the total bone volume of mandibular condyle (BV), the bone surface area of mandibular condyle (BS) and percentage of bony tissue of the mandibular condyle (BV/TV) were measured. All these measurements were done bilaterally. 
Table 2. Descriptive statistics of variables and right / left joint differences

\begin{tabular}{|c|c|c|c|c|c|c|c|}
\hline & \multicolumn{3}{|c|}{ Right } & \multicolumn{4}{|c|}{ Left } \\
\hline & Mean \pm SD & $95 \% \mathrm{Cl}$ & Min-Max & Mean \pm SD & $95 \% \mathrm{Cl}$ & Min-Max & $\mathbf{P}$ \\
\hline MCW & $16.68 \pm 2.55$ & $16.17-17.18$ & $11.38-23.54$ & $16.43 \pm 2.54$ & 15.93-16.94 & $10.43-25.29$ & 0.232 \\
\hline CONM-MF & $4.50 \pm 1.45$ & $4.21-4.79$ & $2-10$ & $4.47 \pm 1.36$ & $4.2-4.74$ & $2-8.01$ & 0.809 \\
\hline CONL-MF & $6.86 \pm 1.85$ & $6.49-7.23$ & $3.2-11.6$ & $6.67 \pm 2.35$ & $6.2-7.14$ & $2-12.8$ & 0.323 \\
\hline CONT-MF(C) & $3.07 \pm 1.21$ & $2.83-3.31$ & $1-8.8$ & $3.19 \pm 1.15$ & $2.97-3.42$ & $1.6-6.4$ & 0.288 \\
\hline CLA-HA & $13.93 \pm 5.11$ & $12.91-14.94$ & $3.42-24.86$ & $14.21 \pm 5.81$ & $13.06-15.36$ & $2.5-34.99$ & 0.646 \\
\hline RLA-VA & $10.66 \pm 3.78$ & $9.91-11.41$ & 3.29-19.61 & $9.31 \pm 3.62$ & $8.59-10.03$ & 2.68-20.82 & $0.001 *$ \\
\hline CLA-RLA & $91.09 \pm 12.23$ & $88.67-93.52$ & $32.66-106.1$ & $92.58 \pm 9.64$ & $90.66-94.49$ & $38-114.21$ & 0.262 \\
\hline CONT-MF(S) & $3.34 \pm 1.16$ & $3.11-3.57$ & $1-8$ & $3.51 \pm 1.28$ & $3.26-3.76$ & $1.6-7.21$ & $0.086 *$ \\
\hline CONMi-CONT & $3.60 \pm 0.88$ & $3.42-3.77$ & $2-6$ & $3.65 \pm 0.95$ & $3.46-3.84$ & $2-8.01$ & 0.531 \\
\hline CONMi-MF & $6.97 \pm 1.53$ & $6.66-7.27$ & 3.6-12 & $7.16 \pm 1.77$ & $6.81-7.51$ & $4-13.61$ & 0.162 \\
\hline AT Tilt & $55.05 \pm 22.74$ & $50.54-59.56$ & 20.73-157.75 & $50.38 \pm 25.24$ & $45.38-55.39$ & $4.51-157.75$ & $0.001^{*}$ \\
\hline AT Height & $4.34 \pm 1.22$ & $4.09-4.58$ & $1.5-8.5$ & $4.27 \pm 1.26$ & $4.02-4.52$ & $1.7-9.6$ & 0.569 \\
\hline AT-ZA Angle & $98.33 \pm 12.34$ & $95.88-100.78$ & $69.03-129.68$ & $97.75 \pm 12.46$ & $95.28-100.22$ & 70.09-126.93 & 0.596 \\
\hline AT-MF & $9.53 \pm 1.96$ & $9.14-9.92$ & $3.7-13.4$ & $9.60 \pm 2.05$ & $9.19-10$ & $5.1-15.3$ & 0.701 \\
\hline TV & $1580.41 \pm 927.60$ & $1396.35-1764.46$ & 238.15-4181.21 & $1592.24 \pm 982.24$ & $1397.35-1787.14$ & $221.76-4105.73$ & 0.672 \\
\hline BV & $884.52 \pm 557.18$ & 773.97-995.08 & $118.2-2550.9$ & $882.87 \pm 592.28$ & $765.35-1000.39$ & $114.08-3651.25$ & 0.946 \\
\hline $\mathrm{BV} / \mathrm{TV}$ & $56.94 \pm 14.42$ & $54.08-59.8$ & $22.56-85.47$ & $57.48 \pm 16.28$ & $54.25-60.71$ & 24.67-89.13 & 0.568 \\
\hline BS & $1742.19 \pm 817.12$ & $1580.06-1904.33$ & $406.45-4296.4$ & $1696.05 \pm 831.56$ & $1531.06-1861.05$ & $404.14-4367.3$ & 0.136 \\
\hline
\end{tabular}

*Statistically significant $\mathrm{p}<0.05, \mathrm{SD}$ — standard deviation; $\mathrm{Cl}$ — confidence interval; Min-Max — minimum-maximum; MCW — the width of mandibular condyle; CONM-MF — the distance in between the mandibular fossa and medial end of mandibular condyle; CONL-MF — the distance between the mandibular fossa and lateral end of mandibular condyle; CONT$-M F(C)$ - the measurement of the distance between the mandibular fossa and tip of the mandibular condyle from the coronal axis; CLA-HA — the angle in between the long axis of mandibular condyle and horizontal plane; RLA-VA — the angle in between the long axis of ramus of the mandible and vertical plane; CLA-RLA — the angle in between the long axis of mandibular condyle and long axis of ramus of mandible; CONT-MF(S) - the measurement of the distance between the mandibular fossa and tip point of the mandibular condyle from the sagitta plane; CONMi-CONT — the distance between the tip point of mandibular condyle and mid-point of the condyle; CONMi-MF — the distance between the mid-point of mandibular condyle and mandibular fossa; AT Tilt — the curvature of articular tubercle; AT Height — the height of articular tubercle; AT-ZA Angle — the angle between the long axis of articular tubercle and transverse axis of zygomatic arch; AT-MF — the distance between the articular tubercle and mandibular fossa; TV — total tissue volume of mandibular condyle; BV — total bone volume of mandibular condyle; BS — the bone surface area of mandibular condyle; BV/TV — the percentage of the bony tissue of mandibular condyle

\section{Statistical analysis}

The statistical analysis of the study was performed using IBM SPSS Statistics 23.0 (IBM Corporation, Armonk, NY, USA) programme. All the measurements were done by two researchers at different times independently and the reliability of the measurements was compared by the Wilcoxon matched double sample test. The normal distribution of all of the variants was examined using the Kolmogorov-Smirnov test. In descriptive statistics, mean \pm standard deviation and minimum-maximum values were used. Additionally, the related variants' mean values in the universe were given in 95\% confidence interval. The differences between the age groups and according to sex were examined by the two-way ANOVA test. The significance of the measurements between the right and left joints was calculated by variance analysis. The changes in the measurements according to age group were detected with Pearson's correlation coefficient.
In all of the statistical analysis, a p-value less than 0.05 was considered significant.

\section{RESULTS}

The mean age of the patients was $44 \pm 14.403$. No statistically significant differences were observed between the mean ages of the patients according to sex $(p>0.05)$. Additionally, there were no statistically significant differences between the measurements of both researchers $(p>0.05)$

The descriptive statistics of the angles in between the MCA are given in Table 1. In Table $2 \mathrm{MCW}$, CONM-MF, CONL-MF, CONT-MF(C), CLA-HA, RLA-VA, CLA-RLA，CONT-MF(S), CONMi-CONT, CONMi-MF, AT Tilt, AT Height, AT-ZA Angle, AT-MF, TV, BV, BS, $\mathrm{BV} / \mathrm{TV}$ are given.

The values of the parameters of the right and left joints are found to be statistically significant $(p \leq 0.05)$. 
In the examination of the whole parameters of the right and left joints without taking the age and sex into consideration, two of the parameters showed statistical significance. These two parameters were RLA-VA $(p<0.001)$ and AT Tilt $(p<0.001)$ and both were found to be greater on the right joints. Additionally, the CONT-MF(S) showed a statistically significant difference between the joint sides $(p=0.086)$ and was higher on the left-joint side (Table 2).

The results according to sex, without taking the age and joint side into consideration, showed statistically significant differences for 8 parameters. From these parameters, the ones which were found to be higher in males were CONT-MF(C) $(p<0.001)$, CONT-MF(S) $(p<0.001)$, CONMi-MF $(p<0.001), B S(p=0.050)$, AT Tilt $(p=0.05)$, AT Height $(p=0.001)$, and AT-MF $(p=0.006)$. Additionally, in females, the AT-ZA Angle $(p=0.005)$ was found to be higher in value. Table 3 shows the descriptive analysis and comparison of sex and age groups.

After examination of all parameters according to age, without taking sex and joint side into consideration, 10 parameters showed statistically significant differences. $\operatorname{CONT-MF}(C)(p=0.05)$, CONT-MF(S) $(p=0.023)$, CONM-MF ( $p=0.011)$, and CONL-MF ( $p=0.018)$ measurements showed statistically significant differences among the different age groups. On examining the measurements of the angle between the mandibular condyle and mandibular ramus among the age groups, RLA-VA $(p=0.050)$ and CLA-RLA $(p=0.049)$ showed statistically significant differences. In the examination of the volume and area measurements of the MCA among age groups, TV $(p=0.012)$, BS $(p=0.024)$ and BV/TV $(p=0.016)$ showed statistically significant differences. Additionally, AT-MF showed statistically significant differences among age groups $(p=0.035)$ (Table 3 ).

On examination of the effects of age on other variables, CONM-MF (30\%), CONL-MF (30\%), CONT-MF(S) (24\%), BV (36\%), BS (35\%) showed a weak correlation; MCA (15\%), MCW (17\%), RLA-VA (18\%), CONMi-MF (15\%), AT-ZA Angle (18\%), BV (17\%) showed a positive correlation with a weak relation. However, the AT Tilt $(-20 \%)$ and BV/TV $(-27 \%)$ showed a negative correlation with a weak relation.

\section{DISCUSSION}

In this study, 19 clinically important morphometric parameters were examined in 100 anatomically normal patients.

In coronal section measurements, Hilgers et al. [2] measured the MCW with $\mathrm{CBCT}$ and found it to

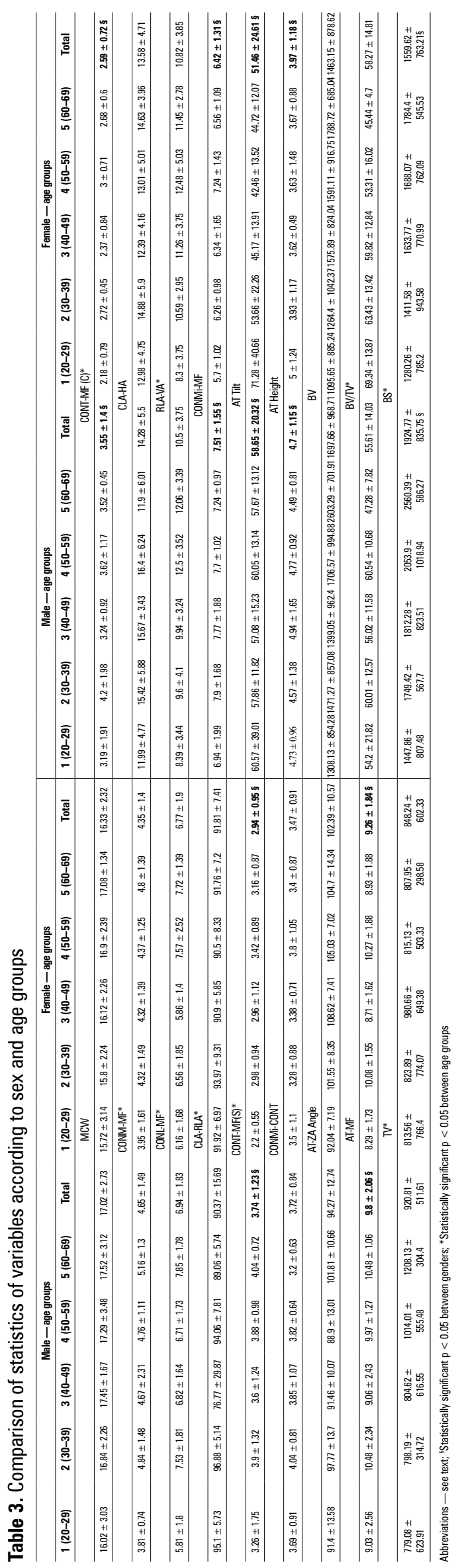


be $18.83 \mathrm{~mm}$. In that study, the authors did not take the sex and joint side into consideration. In the present study, this value was found $16.68 \pm 2.55 \mathrm{~mm}$ on the right side and $16.43 \pm 2.54 \mathrm{~mm}$ on the left side. Ikeda et al. [3] examined the CBCT images of 22 asymptomatic patients of ages in between 12 and 25 (10 males, 12 females). In this study, the measurement of the distance in between the tip of the mandibular condyle and mandibular fossa was measured from the coronal axis. The authors found the measurement values to be $2.6 \pm 0.4 \mathrm{~mm}$ in males and $2.7 \pm 0.6 \mathrm{~mm}$ in females. Additionally, they found no statistically significant difference among the sex groups ( $p>0.05)$. In the current study, a statistically significant difference was observed in the sex groups when the age had not been taken into consideration $(p<0.001)$. The statistically significant difference observed in the present study may be related to the higher number of anatomically normal patients $(p=0.05)$. Ueki et al. [13] performed the measurements of TMJ before and 1 year after sagittal split ramus osteotomy. In patients having symmetrical joint disorders, on the deviated side, the coronal section measurements were found to be $12.7 \pm 13.8^{\circ}$ for CLA-HA before the operation and $12.3 \pm 11.1^{\circ} 1$ year after the surgery. CONM-MF was $1.4 \pm 0.6 \mathrm{~mm}$ before and $1.5 \pm 0.6 \mathrm{~mm}$ 1 year after the surgery. The distance between the mandibular fossa and tip of mandibular condyle was found $1.8 \pm 0.6 \mathrm{~mm}$ before and $2.1 \pm 0.7 \mathrm{~mm}$ after surgery. In another study, Ueki et al. [12] examined the changes in condyle angle after the intraoral vertical ramus osteotomy in 29 patients with prognathism. In preoperative measurements, CLA-HA was found $10.3 \pm$ $\pm 11.4^{\circ}$ on the deviation side and in postoperative measurements, it was found $13.7 \pm 12.7^{\circ}$. The values of the angle measurements of this study were very close to the present study. However, the distances were different. This difference in the present study may be related to the examination done in anatomically normal patients and population differences.

In Zhang et al.'s [15] preliminary study, in coronal sections, nearly similar parameters were examined. The researchers found that the coronal condylar angle (CLA-HA) was $12.05^{\circ}$ on the right and $11.80^{\circ}$ on the left side in the normal population. Additionally, they measured the coronal condyle width of $17.60 \mathrm{~mm}$ on the right and $18.99 \mathrm{~mm}$ on the left side. Both of these parameters did not show any statistically significant difference in their study, and this was in accordance with the present study's findings.
The RLA-VA and CLA-RLA parameters are basically defined in the current study and RLA-VA showed a statistically significant difference in between the right and left joints. This difference may have an importance in the biomechanics of the joint and force distribution through the joint.

Ghristiansen et al. [1] examined the TMJ in 36 healthy patients by $\mathrm{CT}$. In these measurements, they found the distance in between CONT-MF(S) and the joint cavity to be $2.3 \pm 0.9 \mathrm{~mm}$ and AT Tilt $59.6 \pm$ $\pm 12.4^{\circ}$ in the sagittal sections. Similar to the study of Ghristiansen et al. [1], in the current study the authors measured the distance from the tip of the mandibular condyle in the sagittal axis and observed a statistically significant difference between the two sexes $(p<0.001)$. Additionally, in the comparison of the first age group with others, statistically significant differences were observed ( $p=0.023)$. In the current study, AT Tilt showed a statistically significant difference in between right and left sides $(p<0.001)$ and this finding was different from the results of Ghristiansen et al. [1]. In another study, Paknahad et al. [8] measured the mandibular fossa in 40 patients with TMJ disorders (TMD) and 23 controls. They observed the AT Tilt mandibular fossa depth (CONMi-MF) higher in patients with TMD $(p<0.05)$. Different from the study of Paknahad et al. [8], in the present study, the authors examined that parameter without taking the side of the joint into consideration and found a statistically significant difference between the two sexes $(p<0.001)$. Sümbüllü et al. [11] examined the morphology of articular tubercle in 52 patients with TMD and compared it to that in 41 healthy patients in CBCT. Different from the findings of Sümbüllü et al. [11], AT Tilt angle showed a statistically significant difference according to sides $(p<0.001)$ and sexes $(p=0.05)$ in the current study. This main difference in between the two studies is explained by the current study's high number of patients.

In measurements done in sagittal sections, the CONMi-CONT is basically described in the present study. Additionally, the bone surface area was firstly measured in the present study and this parameter was important when evaluated with the other parameters.

AT Tilt has an importance in the movement of mandibular condyle in mandibular fossa and TMD. This value showed a statistically significant difference in between right and left sides in the current study. Additionally, the RLA-VA $(p<0.001)$ value was higher on the right side 
in the current study, which may have been important for the biomechanics of the TMJ and force distribution.

In the literature, there are only a few studies comparing the CONT-MF(C), CONT-MF(S), and CONMi-MF measurements between the sexes $[1,3,8]$. In the present study, all of these values were found to be higher in males in large series comparisons. Additionally, in the present study, BS, AT Tilt, AT Height, AT-MF also showed higher values in males. The current study morphometric measurements related to articular tubercle will be important for the future studies. Knowing the value of AT-MF in normal population will also be a new finding for the zygomatic arch traumas. The AT-ZA Angle was firstly described in this study and it was higher in females when compared with males. This parameter will be important for patients who need long implants to the zygomatic bone.

When all of the parameters are discussed according to age groups only, CONT-MF(C) $(p=0.05)$, CONT-MF(S) $(p=0.023)$, CONM-MF $(p=0.011)$, and CONL-MF $(p=0.018)$ showed a statistically significant difference. Although there are some studies in the literature that compare these parameters $[1,3,13$, 15], none of them made the comparison between age groups. When comparing the angle in between mandibular condyle and mandibular ramus between the age groups, the RLA-VA $(p=0.050)$ and CLA-RLA $(p=0.049)$ showed a statistically significant difference. Although there are some studies that compare these parameters $[12,13]$, none of them compared these parameters between the age groups. In the comparison of the bone volume and surface measurements between the age groups, TV $(p=0.012)$, BS $(p=0.024)$, and BV/TV $(p=0.016)$ showed a statistically significant difference. In the literature, there were no studies examining the TV, BV, BS and BV/TV according to side, age, and sex groups. AT-MF showed a statistically significant difference between the age groups $(p=0.035)$.

In the present study, the examined parameters are detected according to age and these values will support the Moss' functional matrix theory [5].

The obtained values in many parameters of the healthy population and their relations with each other will also have an importance in 3D modelling.

\section{CONCLUSIONS}

The characteristics of TMJ according to age, sex, and joint side must be well known in a healthy popu- lation, in order to understand TMD better. The current study will help understand the aetiology of TMD, the effects of surgical interventions on the joint, the effects of secondary diseases on the joint, the effects of head and neck surgery on TMJ, and the effects of aging on this joint can best be explained.

\section{REFERENCES}

1. Ghristiansen E, Chan T, Thompson J, et al. Computed tomography of the normal temporomandibular joint. Eur J Oral Sci. 1987; 95(6): 499-509, doi: 10.1111/j.1600-0722.1987.tb01966.x.

2. Hilgers ML, Scarfe WC, Scheetz JP, et al. Accuracy of linear temporomandibular joint measurements with cone beam computed tomography and digital cephalometric radiography. Am J Orthod Dentofacial Orthop. 2005; 128(6): 803-811, doi: 10.1016/j.ajodo.2005.08.034, indexed in Pubmed: 16360924.

3. Ikeda K, Kawamura A, Ikeda R. Assessment of optimal condylar position in the coronal and axial planes with limited conebeam computed tomography. J Prosthodont. 2011; 20(6): 432-438, doi: 10.1111/j.1532-849X.2011.00730.x, indexed in Pubmed: 21689204.

4. Kuhn JL, Goldstein SA, Feldkamp LA, et al. Evaluation of a microcomputed tomography system to study trabecular bone structure. J Orthop Res. 1990; 8(6): 833-842, doi: 10.1002/jor.1100080608, indexed in Pubmed: 2213340.

5. Moss ML, Salentijn L. The primary role of functional matrices in facial growth. Am J Orthod. 1969; 55(6): 566-577, indexed in Pubmed: 5253955.

6. Okeson JP. Management of temporomandibular disorders and occlusion. 6 edn. Elsevier Health Sciences, St. Louis 2008.

7. Orhan K, Seki U, Rozylo-Kalinowska I. Diagnostic accuracy of magnetic resonance imaging and clinical signs of temporomandibular joint disorders: a 10-year research update review. Oral Radiology. 2017; 33(2): 81-91, doi: 10.1007/s11282-017-0278-8.

8. Paknahad M, Shahidi S, Akhlaghian M, et al. Is mandibular fossa morphology and articular eminence inclination associated with temporomandibular dysfunction? J Dent (Shiraz). 2016; 17(2): 134-141, indexed in Pubmed: 27284559.

9. Sargon MF. Anatomi akıl notları. Güneş Tıp Kitapevleri 2016: Ankara.

10. Standring S. Gray's anatomy: the anatomical basis of clinical practice. Elsevier Health Sciences 2015.

11. Sümbüllü MA, Cağlayan $F$, Akgül HM, et al. Radiological examination of the articular eminence morphology using cone beam CT. Dentomaxillofac Radiol. 2012; 41(3): 234-240, doi: 10.1259/ dmfr/24780643, indexed in Pubmed: 22074873.

12. Ueki K, Hashiba $Y$, Marukawa $K$, et al. The effects of changing position and angle of the proximal segment after intraoral vertical ramus osteotomy. Int J Oral Maxillofac Surg. 2009; 38(10): 1041-1047, doi: 10.1016/j.ijom.2009.04.021, indexed in Pubmed: 19477622.

13. Ueki $K$, Moroi A, Sotobori $M$, et al. Changes in temporomandibular joint and ramus after sagittal split ramus osteotomy in mandibular prognathism patients with and without asymmetry. J Craniomaxillofac Surg. 2012; 40(8): 821-827, doi: 10.1016/j. jcms.2012.03.003, indexed in Pubmed: 22507292.

14. Winkler S, Dalkowski K, Mair J, Klebe S, Waschke J, Böckers TM, Paulsen F. Sobotta lehrbuch anatomie. Elsevier Health Sciences Germany 2015.

15. Zhang YL, Song JL, Xu XC, et al. Morphologic analysis of the temporomandibular joint between patients with facial asymmetry and asymptomatic subjects by $2 \mathrm{D}$ and $3 \mathrm{D}$ evaluation: a preliminary study. Medicine (Baltimore). 2016; 95(13): e3052, doi: 10.1097/ MD.0000000000003052, indexed in Pubmed: 27043669. 